\title{
Cloud Based Material Management System for Printing Press
}

\author{
Yongbin Zhang \\ Department of Mechanical Engineering \\ Beijing Institute of Graphic Communication \\ Beijing, China \\ zhangyongbin@bigc.edu.cn
}

\section{Ronghua Liang}

Department of Teaching Technology and Network

Beijing Institute of Graphic Communication

Beijing, China

liangronghua@bigc.edu.cn

\author{
Yanping Du \\ Department of Mechanical Engineering \\ Beijing Institute of Graphic Communication \\ Beijing, China \\ duyanping@bigc.edu.cn \\ Li Wei \\ Department of Mechanical Engineering \\ Beijing Institute of Graphic Communication \\ Beijing, China \\ weili@bigc.edu.cn
}

\begin{abstract}
Material management is one of the most important factors affecting printing companies' profits in China. Competition has been intensified in traditional printing market. More and more printing companies need innovational methods to improve printing management in order to keep their competition. We analyzed material management problems in printing companies and presented a cloud based material management to manage printing material effectively and efficiently. We focused on managing raw material within printing company to simplify the prototype system. Business process management method was used to identify material management related processes. Business process model and notation was adapted to model and express identified processes. A printing material management prototype system was designed and deployed with Software as a Service model. Results showed this model could reduce cost for printing management and help printing companies manage the raw material effectively and efficiently. Much work will focus on extending the scope of material management in the future.
\end{abstract}

Keywords-material management;business process management; cloud computing;Software as a Service; printing

\section{INTRODUCTION}

Competition among printing companies is fiercer than ever in China. On one hand, the costs of printing have been increasing, especially for costs of paper and manual works. Too many printing companies is the another reason for the increasing competition [1]. For the past decade, the price for the same printing order keeps almost the same but the manual cost has doubled, which means the profit for printing companies has been deduced largely. On the other hand, the rapid development of digital devices and network, especially the Internet and mobile devices, more and more information is available in digital format instead of paperprinted format. This reduces the requirement of traditional printing.

Innovation is the key for printing plants to survive current competition. There are more than 100,000 printing companies and 95 percent of them are private-owned ones [2]. Big companies have adopted new information technology to enhance their competition. These companies have use enterprise resource planning (ERP) systems to manage all information [3], including customer information, employee, order, finance and so on. More than 95 percent printing companies in China have used computers in their daily work according to our surveys. More than 80 percent companies in Beijing have used ERP to manage their information.

Information technologies have not achieved expected results in most printing companies. Most printing companies just replaced the daily paper working documents with digital ones. Workers think it is more convenient to send and copy digital documents than paper ones. At the same time, they face problems with digital documents, such as outdate documents, inconsistence among related documents and version controlling problem.

Printing Material is the most valuable part of a printing order. Paper, link, plates and other printing related material costs more than 70 percent of entire cost of an order. Effect and efficient management of printing material will reduce wasting and will help printing company to reduce cost and to increase competition.

In this paper, we will provide a cloud based printing material management system framework. With this framework, printing plants can manage their material effectively without extra investment on hardware and software system.

\section{MATERIALS MANAGEMENT}

Materials management is part of business logistics and refers to overseeing the location and movement of physical items or products. There are three main elements associated with such management including spare parts, quality control, and inventory management [4].

The first element in materials management involves spare parts at a business. A detailed business process is required to determine the order point for spare parts, 
identify the ideal quantity to order, and process receipt of the parts to make sure they are in the correct place. Spare parts are integral to the continuing operation of production lines and related equipment. Poor management of this process can cause downtime and loss of production as machines break down without the parts available to repair them.

Quality control, ensuring products are of high and consistent value is a major part of materials management. The creation of material standards, inspections, and a returns process is a primary responsibility of employees at a company. All parts and materials must be tested to ensure that a specific level of quality is met. This is typically completed before a payment is issued to a supplier, ensuring that the supplier has met the conditions of their contract.

Inventory management is the accurate tracking of all materials in a company's inventory. A company has typically purchased these items from another supplier. There are three possible areas of loss that are reduced through effective inventory management: shrinkage, misplacement, and short shipments.

Shrinkage is a general materials management term used to describe the loss of materials once they have reached a company. This type of loss can be due to theft or damage. Loss through misplacement is most commonly found in very large organizations or warehouses. Material is received by the shipper and then frequently moved to another location by the distribution staff. However, if it is moved to the wrong location, it can become lost and counted as never having been received.

Short shipments occur when the quantity received is less that the number on the packing slip. This must be identified and corrected as soon as possible, preferably before the shipper receives the package. The more time that passes before it is realized, the greater is the risk of a supplier insisting that the product was shipped correctly, and the loss occurred within the customer's warehouse.

Materials management is important in large manufacturing and distribution environments, such as warehouses, where there are multiple parts, locations, and significant money invested in these items.

\section{LITERATURE VIEW}

More and more researchers have realized that information technology will be the key for success of traditional printing and press industry. With the increment of digital information and smart devices, traditional paperprinted documents are decreasing. Traditional printing and press companies have faced to the fact of declining business. Information technologies have been used in many aspects of printing, e.g. improving printing quality and producing management.

In China, most printing companies still focus on printing related devices. About 95 percent of Chinese printing companies are private ones. If a printing device goes out of work, the ongoing work have to be stopped. The owner of the companying can see the direct effect. On the other hand, lack finance support is one characteristic of private company. It is difficult to calculate the return of the investment on information technology. It is natural for private company owner focusing on the printing related devices.
Enterprise Resource Management (ERP) system is one of the most populated systems for make-to-order companies [5]. At present, there are 50 percent of printing companies have adopted ERP. There are more than 100 printing ERP systems in China. The prices of ERP range from less than 10,000 RMB to more than 1,000,000 RMB.

Job Definition Format (JDF) specification gets more focus recently. JDF is drafted by The International Cooperation for the Integration of Processes in Prepress, Press, and Postpress Organization (CIP4). The mission of CIP4 is to foster the adoption of process automation in the printing industry. JDF specification has three main functions including providing a single common language that supports the lifecycle of a print job, providing a command and control language for devices on the shop floor and providing a flexible methodology for constructing workflows and providing the command, control, and configuration of plant automation and job production [6].

JDF can be used in printing ERP systems. Some researchers discuss JDF based ERP system [7]. This effort helps to combine job information and production information and to bring benefits for precious management.

Most of printing companies in china are Small and medium size. There were more than 100 thousand printing companies by the end of 2012 in China. Most of them were small and medium size ones. For example, there were 1691 printing companies in Beijing in 2012. There were only 92 companies whose yearly incomes were greater than 50 million YUAN in RMB [8].

Cost of printing material comprises from 55 percent to 88 percent of the entire cost of a printing order. Effectively and efficiently managing printing material will help reduce the cost of a printing job. At present, ERP systems provide information management methods for printing companies. ERP provide many functions such as order management, client management and material management. But ERP have not achieved the expected results. There are many causes leading to this result. The first one is that management in printing company should be enhanced. ERP system will not be effective if management in a company does not match the one embedded in the ERP system. The second reason is that employees need more training for using ERP systems. Without participation of employees, ERP system does not achieve the expected effect. The third reason is that investment on hardware successfully running ERP system is low. The fourth one is that ERP systems are too complex for employees to use effectively.

Material management is an isolated system in most small-medium size printing companies. Printing company has realized that material management is key factor for business success. Material information was written on paper in the past few decades. At present, with the commonly use of personal computer, most of printing companies adopted material management system or electronic documents such as office excel or word documents. Material information management system helps printing companies to manage material conveniently. Even for printing companies who use office documents to manage material, it is more convenient to search and find material information with electronic documents than with paper ones. 
To solve the problems mentioned above. We focused on material management. We design a printing material management. With cloud computing technology, we provide the system as a service so that printing companies can use the system without investment on the software system and the hardware running the system as traditional one. Printing companies do not need IT stuff to maintain the system.

\section{METHODS}

\section{A. Business Process Management}

Business process management (BPM) is a systematic approach to making an organization's workflow more effective, more efficient and more capable of adapting to an ever-changing environment [9]. A business process is an activity or set of activities that will accomplish a specific organizational goal.

BPM is a way of looking at and then controlling the processes that are present in an organization. It is an effective methodology to use in times of crisis to make certain that the processes are efficient and effective, as this will result in a better and more cost efficient organization.

The goal of BPM is to reduce human error and miscommunication and focus stakeholders on the requirements of their roles. BPM is a subset of infrastructure management, an administrative area concerned with maintaining and optimizing an organization's equipment and core operations.

BPM is often a point of connection within a company between the line-of-business (LOB) and the IT department. Business Process Execution Language (BPEL) and Business Process Management Notation (BPMN) were both created to facilitate communication between IT and the LOB. Both languages are easy to read and learn, so that business people can quickly learn to use them and design processes. Both BPEL and BPMN adhere to the basic rules of programming, so that processes designed in either language are easy for developers to translate into hard code.

There are three different kinds of BPM frameworks available in the market today. Horizontal frameworks deal with design and development of business processes and are generally focused on technology and reuse. Vertical BPM frameworks focus on a specific set of coordinated tasks and have pre-built templates that can be readily configured and deployed. Full-service BPM suites have five basic components: Process discovery and project scoping, Process modeling and design, Business rules engine, Workflow engine and Simulation and testing.

Business process management plays a central role at operational, organizational and technological levels. A company's competitiveness relies heavily on its business processes, and thus the need to execute business processes with agility and efficiency is ever-increasing.

Business process management method can be used to manage printing material process. Business processes are the fundamental building blocks of an organization's success. A business process explicitly expresses activities order, input, output, logical and executor. Most printing companies do not explicitly define their material management process. Some printing companies do not use information technology to manage material information. Some others may have material management information system but the problem is that information in the system does not match material entity. The existing system does not help printing company as expected. One of the main causes is that information in the system is not updated timely.

With business process management method, printing material related processes were explicitly defined. Store and remove material are two key processes in printing material management. So material procurement management process and material delivery management process are two important business processes. With these two processes, the procured material information can be inputted into the system after quality and quantity checking then material information can be updated when removing from stock.

\section{B. Business Process Model and Notation}

Process modeling produces abstract descriptions of business processes that are a central asset to the organization as they enable its specification, documentation, analysis and engineering through multiple paradigms, languages and techniques. Business Process Model and Notation specification is used to describe printing material process.

Business Process Model and Notation (BPMN) is a method of illustrating business processes in the form of a diagram similar to a flowchart. BPMN was originally conceived and developed by the Business Process Management Initiative (BPMI). It is currently maintained by the Object Management Group (OMG).

BPMN provides a standard, easy-to-read way to define and analyze public and private business processes [10]. BPMN provides a standard notation that is readily understandable by management personnel, analysts and developers [11]. The original intent of BPMN was to help bridge communication gaps that often exist between the various departments within an organization or enterprise. BPMN can also help to ensure that XML (Extensible Markup Language) documents designed for the execution of diverse business processes can be visualized with a common notation.

A standard Business Process Model and Notation (BPMN) will provide businesses with the capability of understanding their internal business procedures in a graphical notation and will give organizations the ability to communicate these procedures in a standard manner. Furthermore, the graphical notation will facilitate the understanding of the performance collaborations and business transactions between the organizations. This will ensure that businesses will understand themselves and participants in their business and will enable organizations to adjust to new internal and $\mathrm{B} 2 \mathrm{~B}$ business circumstances quickly.

A diagram in BPMN is assembled from a small set of core elements, making it easy for technical and nontechnical observers to understand the processes involved. Elements are categorized into three major groups called flow objects, connecting objects and swim lanes. Flow objects, denoted by geometric figures such as circles, rectangles and diamonds, indicate specific events and activities. Flow objects are linked with connecting objects, which appear as solid, dashed or dotted lines that may include arrows to indicate process direction. Swim lanes 
are denoted as solid, straight lines running lengthwise within a rectangle called a pool. The swim lanes organize diverse flow objects into categories having similar functionality.

\section{Software-as-a-Service}

Software as a Service (SaaS) model of cloud computing technology is used to deploy the printing management system. With SaaS model, printing companies can access the printing material management system from anywhere at any time with web browser if internet connection is available [12]. Printing companies do not need to maintain the hardware and running system.

Cloud computing is a general term for anything that involves delivering hosted services over the Internet. These services are broadly divided into three categories: Infrastructure-as-a-Service (IaaS), Platform-as-a-Service $(\mathrm{PaaS})$ and Software-as-a-Service (SaaS). The name cloud computing was inspired by the cloud symbol that's often used to represent the Internet in flowcharts and diagrams.

A cloud service has three distinct characteristics that differentiate it from traditional hosting. It is sold on demand, typically by the minute or the hour; it is elastic -a user can have as much or as little of a service as they want at any given time; and the service is fully managed by the provider (the consumer needs nothing but a personal computer and Internet access). Significant innovations in virtualization and distributed computing, as well as improved access to high-speed Internet and a weak economy, have accelerated interest in cloud computing.

A cloud can be private or public. A public cloud sells services to anyone on the Internet. Currently, Amazon Web Services is the largest public cloud provider. A private cloud is a proprietary network or a data center that supplies hosted services to a limited number of people. When a service provider uses public cloud resources to create their private cloud, the result is called a virtual private cloud. Private or public, the goal of cloud computing is to provide easy, scalable access to computing resources and IT services.

Infrastructure-as-a-Service like Amazon Web Services provides virtual server instance API to start, stop, and access and configure their virtual servers and storage. In the enterprise, cloud computing allows a company to pay for only as much capacity as is needed, and bring more online as soon as required. Because this pay-for-what-youuse model resembles the way electricity, fuel and water are consumed. It's sometimes referred to as utility computing.

Platform-as-a-service in the cloud is defined as a set of software and product development tools hosted on the provider's infrastructure. Developers create applications on the provider's platform over the Internet. PaaS providers may use APIs, website portals or gateway software installed on the customer's computer. Force.com and GoogleApps are examples of PaaS. Developers need to know that currently, there are not standards for interoperability or data portability in the cloud. Some providers will not allow software created by their customers to be moved off the provider's platform.

In the software-as-a-service cloud model, the vendor supplies the hardware infrastructure, the software product and interacts with the user through a front-end portal. SaaS is a very broad market. Services can be anything from
Web-based email to inventory control and database processing. Because the service provider hosts both the application and the data, the end user is free to use the service from anywhere.

While on-premise business process management (BPM) has been the norm for most enterprises, advances in cloud computing have leaded to increased interest in on-demand, software as a service (SaaS) offerings.

\section{A CASE STUDY}

We set two principles when we designed the cloud based printing material management system. Efficiency was the first principle. We did not start from scratch. We would not build the business process engine and would not build the Platform as a service by ourselves. Focusing on core printing material business processes was another principle. There are many different processes for even a small size printing company. It was impossible to design all related process in a short time.

We investigated the printing factory in Beijing Institute of Graphic Communication (BIGC) and designed the system prototype. There are many reasons to choose the printing factory in BIGC. First, board band Internet access has been available for the printing factory of BIGC for a long time while other printing factories, especially small and medium size ones, have local area network instead of wide area network. Second, the printing factory of BIGC is a small-sized company and related material management processes are simple. Third, it is very convenient for us to access and investigate the printing requirement.

There were two important processes related with material management. One was material procurement process and the other was printing order management process. All material was used during one of prepress, press and post press processes. The need for any related material was processing during the execution of an ordering as shown in Fig .1.

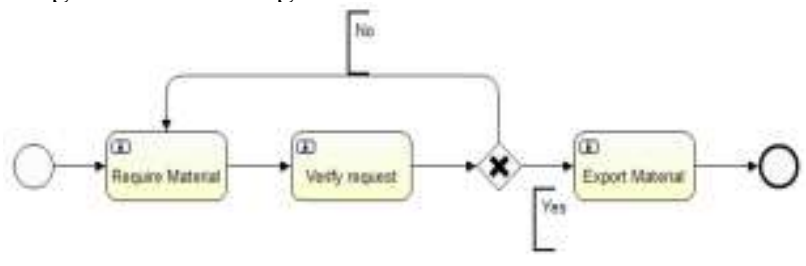

Figure 1. Material consuming process

The material procurement and material quality checking process was shown in Fig .2.

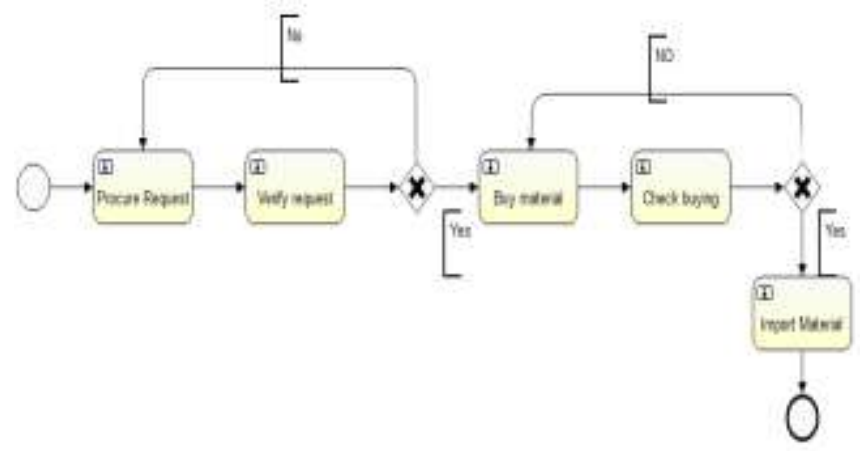

Figure 2. Material Procurement process. 
An open source business process engine named Activiti was used as business process engine. Activiti is implemented in Java programming and provides flexible application programming interfaces [13].

A free and restricted Platform as a service named Cloudbees was used as PaaS to deploy our designed printing management system. Cloudbees mainly support different web servers for Java programming language [14]. It reduced the learning cures for cloud computing for java web developers. We designed a printing material management web system with java language and deployed the system on Cloudbees.

Our designed printing management system provided as a material management service for BIGC printing factory. With explicitly expressed material management processes, users could understand the processes better. Material information could be updated instantly. Employees could access the system from anywhere at any time from personal computer, smart mobile phone or tablet as long as internet access available. The printing factory could reduce cost of system maintenance.

\section{CONCLUSION}

We designed a printing material management system and deployed as Software as a service model. Two core processes related to printing material management were defined with business process management method. The two processes explicitly expressed the material management activities and logics and helped the printing company to understand material management processes better. The processes would provide a base for future material management processes optimization. With cloud computing technology, it would be possible for different sized printing companies to adopt information technology to improve material management efficiency. The value of this paper was that it provided a reference for other printing management applications. The limitation of this research was that the complexity of printing material management was not considered. More work will be done to deal with the complexity of printing material management processes in the future.

\section{ACKNOWLEDGMENT}

Yongbin Zhang thanks Beijing Municipal Commission of Education for the finical support with grant number KM201310015008 and the support with The Importation and Development of High-Caliber Talents Project of Beijing Municipal Institutions.

\section{REFERENCES}

[1] He mingxin, "Printing companies should be leading group going aboard," China Publishing Journal, , vol. 6, June. 2012,pp.19-21.

[2] Ren zhicheng, "Innovation is the key for printing industry development," China Pbulishing Journal, vol. 4, April. 2011,pp.2730.

[3] SUN Liang. "Dyeing and Printing Company Production ERP System Design," Journal of Donghua University(Natural Science),vol. 36, DEC. 2010.pp.708-712.

[4] M Christopher.Logistics and supply chain management,Pearson UK,2012.

[5] Aslan, B., M. Stevenson, and L.C. Hendry, Enterprise Resource Planning systems: An assessment of applicability to Make-ToOrder companies. Computers in Industry, vol. 63,2012.pp. 692-705.

[6] JDF Specification. http://www.cip4.org/document_archive/download_request.php?did $=2591$.

[7] Yun Fei Zhong, Xiao Qi Peng, Xi Yu Xiao, Qiao Li Zhang, Qian Kun Cheng, "A Preliminary Study of JDF-Based ERP System in the Printing Enterprises," Advanced Materials Research, vol. 102104,2010 pp.851-855.

[8] Beijing Printing industry development http://www.bjysxh.org/news/jingyan/2013-04-17-879.html.

[9] John Jeston, Johan Nelis,Business process management,NY:Routledge, 2013.

[10] Bruce Silver ,BPMN Method and Style, 2nd Edition.Cody-Cassidy Press, 2011.

[11] Chinosi, M. and A. Trombetta, BPMN: An introduction to the standard. Computer Standards \& Interfaces, vo1. 34, 2012. pp. 124-134.

[12] Alexander Benlian,Thomas Hess,Peter Buxmann ,Software-as-aService,Springer.2010.

[13] Activiti BPM Platform.http://http://www.activiti.org/

[14] CloudBees Platform.http://www.cloudbees.com/platform. 